\title{
Dry-Contact and Noncontact Biopotential Electrodes: Methodological Review
}

\author{
Yu Mike Chi, Student Member, IEEE, Tzyy-Ping Jung, Senior Member, IEEE, and \\ Gert Cauwenberghs, Senior Member, IEEE
}

Methodological Review

\begin{abstract}
Recent demand and interest in wireless, mobile-based healthcare has driven significant interest towards developing alternative biopotential electrodes for patient physiological monitoring. The conventional wet adhesive $\mathrm{Ag} / \mathrm{AgCl}$ electrodes used almost universally in clinical applications today provide an excellent signal but are cumbersome and irritating for mobile use. While electrodes that operate without gels, adhesives and even skin contact have been known for many decades, they have yet to achieve any acceptance for medical use. In addition, detailed knowledge and comparisons between different electrodes are not well known in the literature. In this paper, we explore the use of dry/noncontact electrodes for clinical use by first explaining the electrical models for dry, insulated and noncontact electrodes and show the performance limits, along with measured data. The theory and data show that the common practice of minimizing electrode resistance may not always be necessary and actually lead to increased noise depending on coupling capacitance. Theoretical analysis is followed by an extensive review of the latest dry electrode developments in the literature. The paper concludes with highlighting some of the novel systems that dry electrode technology has enabled for cardiac and neural monitoring followed by a discussion of the current challenges and a roadmap going forward.
\end{abstract}

Index Terms-Biopotentials, electrocardiograms (ECG), electroencephalograms (EEG).

\section{INTRODUCTION}

B IOPOTENTIAL recordings in the form of electrocardiograms (ECG), electroencephalograms (EEG), electrooculograms (EOG) and electromyograms (EMG) are indispensable and vital tools for both medical and research use. These well-

Manuscript received August 11,2010; accepted September 21,2010. Date of publication October 11, 2010; current version December 08, 2010. This work was supported in part by National Semiconductor, by the National Science Foundation, by the NIH/NIA, and by the Defense Advanced Research Projects Agency.

Y. M. Chi is with the Department of Electrical and Computer Engineering, Jacobs School of Engineering, University of California, San Diego, CA 92093 USA (e-mail: m1chi@ucsd.edu).

T.-P. Jung is with the Swartz Center for Computational Neuroscience, Institute for Neural Computation, University of California, San Diego, CA 92093 USA (e-mail: tpjung@ucsd.edu).

G. Cauwenberghs is with the Department of Bioengineering, Jacobs School of Engineering, and Institute for Neural Computation, University of California, San Diego, CA 92093 USA (e-mail: gert@ucsd.edu).

Color versions of one or more of the figures in this paper are available online at http://ieeexplore.ieee.org.

Digital Object Identifier 10.1109/RBME.2010.2084078 proven signal modalities provide a wealth of physiological information, which by virtue of modern bioinstrumentation technology can be harnessed noninvasively and inexpensively for the emerging global health applications of clinical physiological monitoring and medical treatment [1], [2].

Traditionally, $\mathrm{Ag} / \mathrm{AgCl}$ electrodes with wet conductive gels are used for biopotential recordings. The standard $\mathrm{Ag} / \mathrm{AgCl}$ electrode has been well-characterized and studied over many decades [3]-[5]. Most of its properties are well understood [6], and sufficient empirical data exists for mechanism that are not, such as low-frequency noise and drift [4]. Nevertheless, with proper preparation, the signal is excellent.

The basic principles behind gel-less electrodes are also well known. Despite decades of research in alternative biopotential sensor technologies [7]-[10] for ECG and EEG applications, the standard wet $\mathrm{Ag} / \mathrm{AgCl}$ electrode is still almost universally used for clinical and research applications. Each year billions of disposable adhesive ECG clinical electrodes are produced, while dry electrodes are limited to niche, nonmedical/scientific, applications like fitness monitoring and toys.

The usefulness and performance of dry and noncontact electrodes can be divided in to two categories. The first relates to the to the signal quality of the device in terms of noise and motion sensitivity. Second, because electrodes interface to the skin either in contact or close proximity to the body, the specific electrode must also be evaluated for comfort and utility at the system level. This paper aims to critically address the latest developments in dry and noncontact electrodes accounting for both of these considerations. One chief advantage of the standard clinical wet electrode is the fact that it adheres very well to skin. While problematic from a patient comfort standpoint for long-term use, adhesive wet electrodes stay fixed to specific, clinical-standard locations on the body. Dry and noncontact electrodes address the comfort issues with the adhesive wet electrode, but are much more difficult to secure against the patient. Thus for these technologies to be clinically useful, mechanical solutions must be devised to place the electrodes in the proper position (such as the 12-lead ECG) or an alternative application niche must be found. It is for these reasons, that dry and noncontact electrodes are unlikely to replace the standard hospital ECG or EEG electrode.

The literature around dry electrode technology is quite vast, but dispersed across multiple, semi-isolated, research groups and publications. In addition, the amount of information is compounded by all of the possible applications (ECG, EEG, etc). 

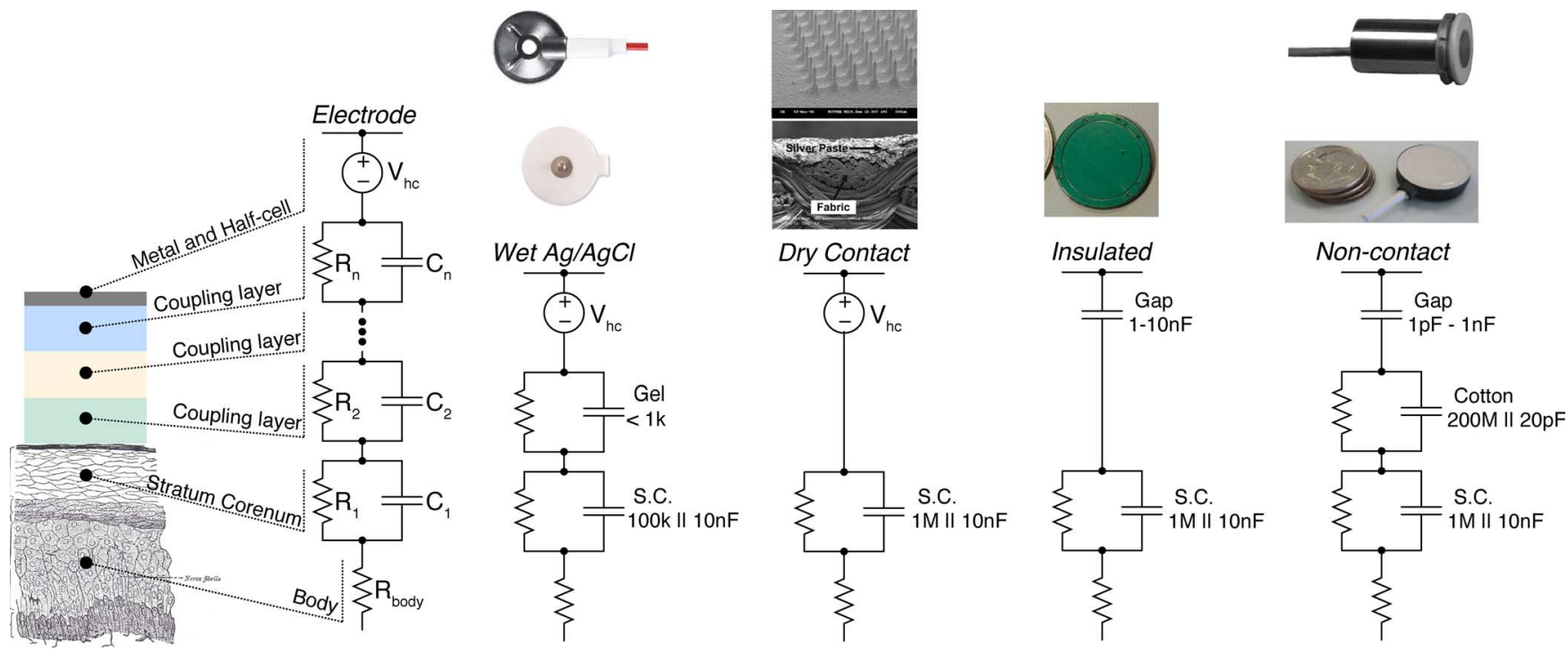

Fig. 1. Electrical coupling of skin-electrode interface for various electrode topologies, including wet-contact gel-based $\mathrm{Ag} / \mathrm{AgCl}, \mathrm{dry}-\mathrm{contact} \mathrm{MEMS}$ and metal plate, thin-film insulated metal plate, and noncontact metal plate coupling through hair or clothing such as cotton. Insets show examples of practical electrodes for each category as described in Section III.

With that in mind, this paper reviews the latest developments in dry/noncontact electrodes while providing a historical context and a discussion of the challenges and future directions for this field. In 2000, Searle et al. [3] published a detailed comparison between standard wet $\mathrm{Ag} / \mathrm{AgCl}$ and their specific implementation of a dry and insulating electrodes from an impedance, interference motion artifact rejection perspective. In contrast to conventional wisdom, their paper demonstrated that dry and insulate electrodes (if buffered and shielded) can perform as well, if not better than, standard wet $\mathrm{Ag} / \mathrm{AgCl}$ electrodes in each of these respects. However, the intrinsic noise properties of the electrode were not discussed and the paper was limited to only two, specific dry and insulated electrode implementations.

This paper presents a systematic comparison between the various contact and noncontact electrode technologies with a focus on quantifying the noise performance and motion sensitivity as a function of physical and electrical parameters, as well as their unobtrusiveness and ease for clinical use. The following section presents a general model of the electrode interface, described and characterized with measurements from an electrical perspective. This establishes the fundamental principles for dry and noncontact electrodes and describes the fundamental signal quality limits. The different electrode technologies and their properties are surveyed next, and the paper concludes with a discussion of the latest developments in the literature along with future directions and challenges.

\section{SKIN-ELECTRODE INTERFACE}

The concept of "electrode" is rooted in the study of electrochemical cells where electrical transport is governed by oxidation and reduction reactions taking place at the interface between a metal and an electrolyte. A conventional wet-contact electrode fits this description, since the metal conductor of the electrode is bathed in an electrolyte gel or solution that buffers the electrolytic composition through the outer and inner layers of the skin. Therefore, a wet-contact electrode is well characterized by a half-cell potential, a double layer capacitance, and parallel and series resistances as shown in Fig. 1. For a dry-contact or noncontact electrode, however, the interface is more complex and other processes enter the electrical interactions in skin-electrode coupling. The performance of the electrode is critical, especially given the small signal amplitude of ECG $(1 \mathrm{mV})$ and EEG signals $(10-100 \mu \mathrm{V})$.

In general, the coupling between skin and electrode can be described as a layered conductive and capacitive structure, with series combinations of parallel $\mathrm{RC}$ elements. The type of electrode and skin coupling results in several such structures, as shown in Fig. 1, with different conductance and capacitance values. For each of these electrode types, typically one of the RC sections dominates and the electrical coupling may be represented as a single element with conductance $g_{c}$ in parallel with capacitance $C_{c}$, or a simplified coupling admittance $Y_{c}(j \omega)=g_{c}+j \omega C_{c}$.

It is important to realize that both conductance and capacitance are important in characterizing electrode performance. In what follows we will show that the conventional notion that low resistance (high conductance) is essential for good electrode performance could be misleading, and that maximizing resistance (minimizing conductance) in electrode-skin coupling is actually beneficial in certain important limiting cases. This unconventional and seemingly counter-intuitive observation derives from simple circuit theory validated by experimental data, which we offer here for the benefit of the reader who may have missed this important point from previous literature coverage on electrode interfaces. Thereby, we hope to rectify misunderstandings in the role of coupling conductance on noise performance and sensitivity to guide better and more informed decisions in the design of the electrode and the skin coupling medium.

\section{A. Electrical Model}

To accurately model the effect of the skin-electrode coupling admittance $Y_{c}(j \omega)$ on the quality and robustness of the received 

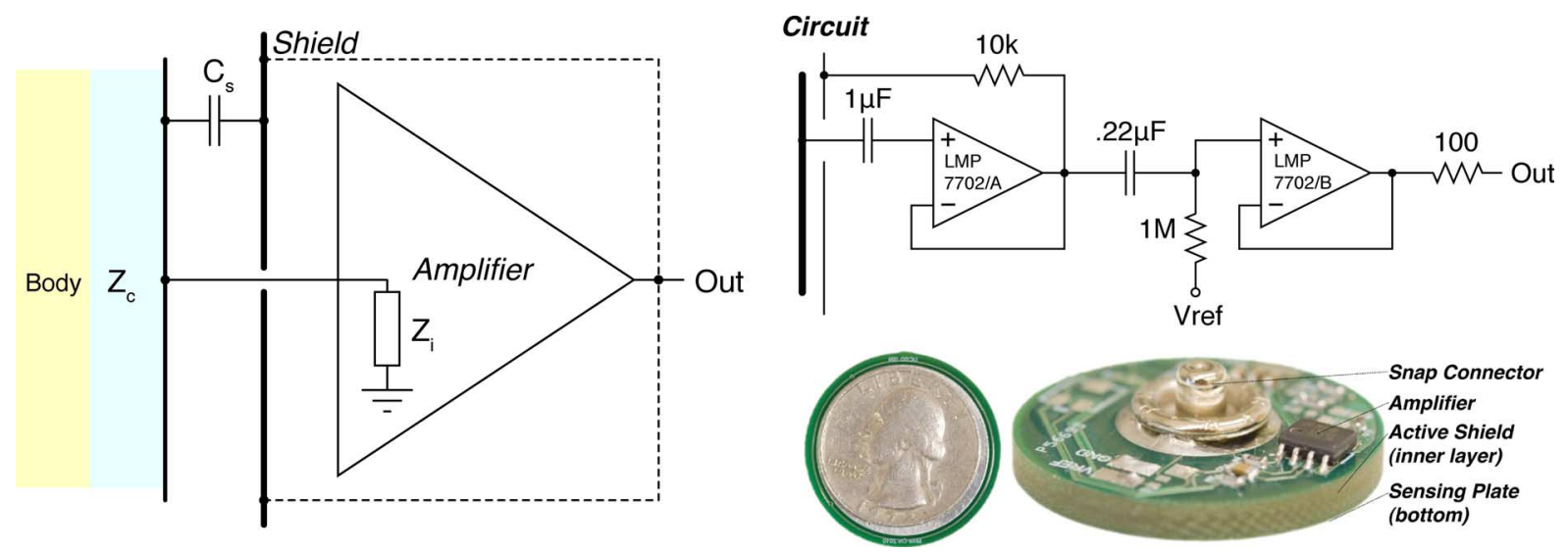

Fig. 2. (Left) Simplified topology and circuit model of a general, actively shielded biopotential amplifier [11]. Active shield guards high-impedance input from interference by other sources and implies capacitive coupling between source and amplifier output. (Right) Simple implementation for dry active electrode made from standard PCB [14]. Exposed metal on bottom surface contacts skin. The electrode can also work as a noncontact through insulation such as cotton. More complex designs can be found in [11]-[13].

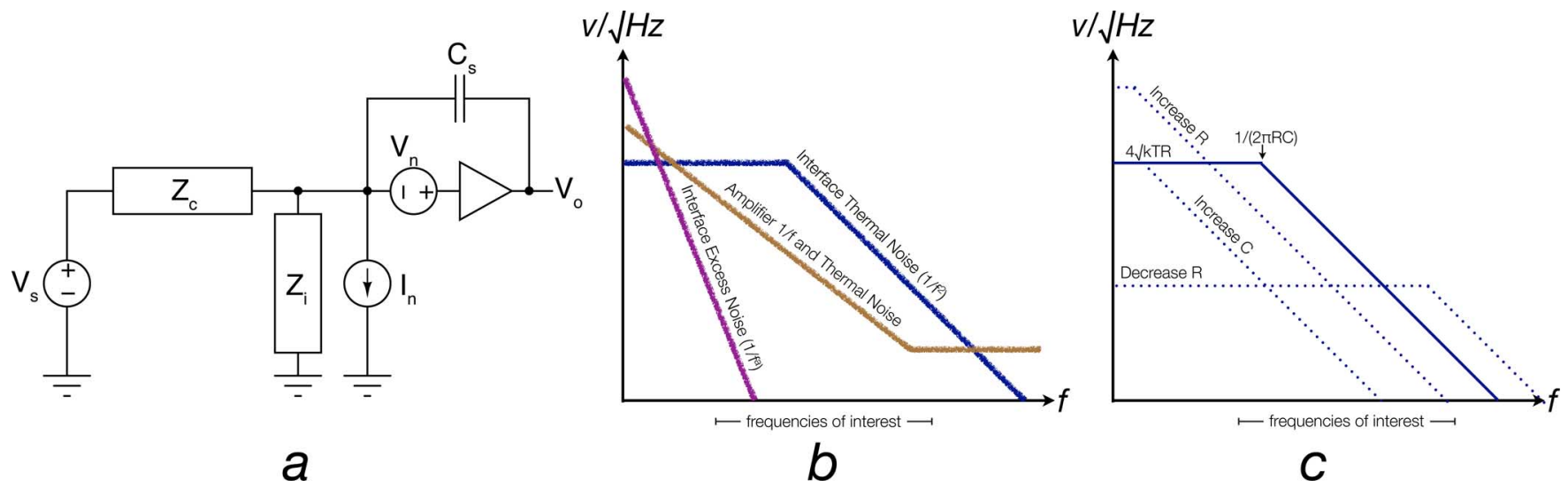

Fig. 3. Dry/noncontact amplifier circuit noise model along (a) with a simplified plot of frequency behavior of (b) various noise sources. (c) For each RC layer, noise contribution can be decreased by either drastically increasing resistance towards infinity, increasing capacitance, or reducing the resistance towards zero.

signal, it is necessary to account for the electrical coupling between the skin and the amplifier connected to the electrode to acquire the signal. We consider the general, actively shielded amplifier topology shown in Fig. 2 (left), chosen for its relative immunity to interference from other sources and line noise [3]. This topology conforms to many of the published amplifier circuits for dry-contact and noncontact electrodes, e.g., [11]-[13]. A particularly simple low-power and compact realization, which is used in the experimental data presented in this survey, is illustrated in Fig. 2 (right).

We define the following electrical signals and parameters in reference to the circuit topology in Fig. 2 (left) and its noise model in Fig. 3 (left):
$v_{s}(j \omega) \quad$ signal source on skin surface;
$v_{o}(j \omega) \quad$ signal recorded at amplifier output;
$v_{i, n}(j \omega) \quad$ input referred amplifier voltage noise;
$i_{i, n}(j \omega) \quad$ net current noise at amplifier input;
$Y_{c}(j \omega) \quad g_{c}+j \omega C_{c}$, skin-electrode coupling admittance;
$Y_{i}(j \omega) \quad g_{i}+j \omega C_{i}$, amplifier input admittance;

$C_{s} \quad$ active shield to electrode capacitance;

$A_{v} \quad$ amplifier voltage gain.

As shown in the Appendix, the resulting received output signal $v_{o}$ can be written as

$$
v_{o}=G(j \omega)\left(v_{s}+v_{s, n}\right)
$$

with a source-to-output signal voltage gain

$$
\begin{aligned}
G(j \omega) & =A_{v} \frac{Y_{c}(j \omega)}{Y_{c}(j \omega)+Y_{i}(j \omega)+j \omega\left(1-A_{v}\right) C_{s}} \\
& =A_{v} \frac{g_{c}+j \omega C_{c}}{g_{c}+g_{i}+j \omega\left(C_{c}+C_{i}+\left(1-A_{v}\right) C_{s}\right)}
\end{aligned}
$$

and source input-referred voltage noise

$$
\begin{aligned}
v_{s, n} & =\frac{Y_{c}(j \omega)+Y_{i}(j \omega)+j \omega C_{s}}{Y_{c}(j \omega)} v_{i, n}+\frac{i_{i, n}}{Y_{c}(j \omega)} \\
& =\frac{g_{c}+g_{i}+j \omega\left(C_{c}+C_{i}+C_{s}\right)}{g_{c}+j \omega C_{c}} v_{i, n}+\frac{i_{i, n}}{g_{c}+j \omega C_{c}}
\end{aligned}
$$



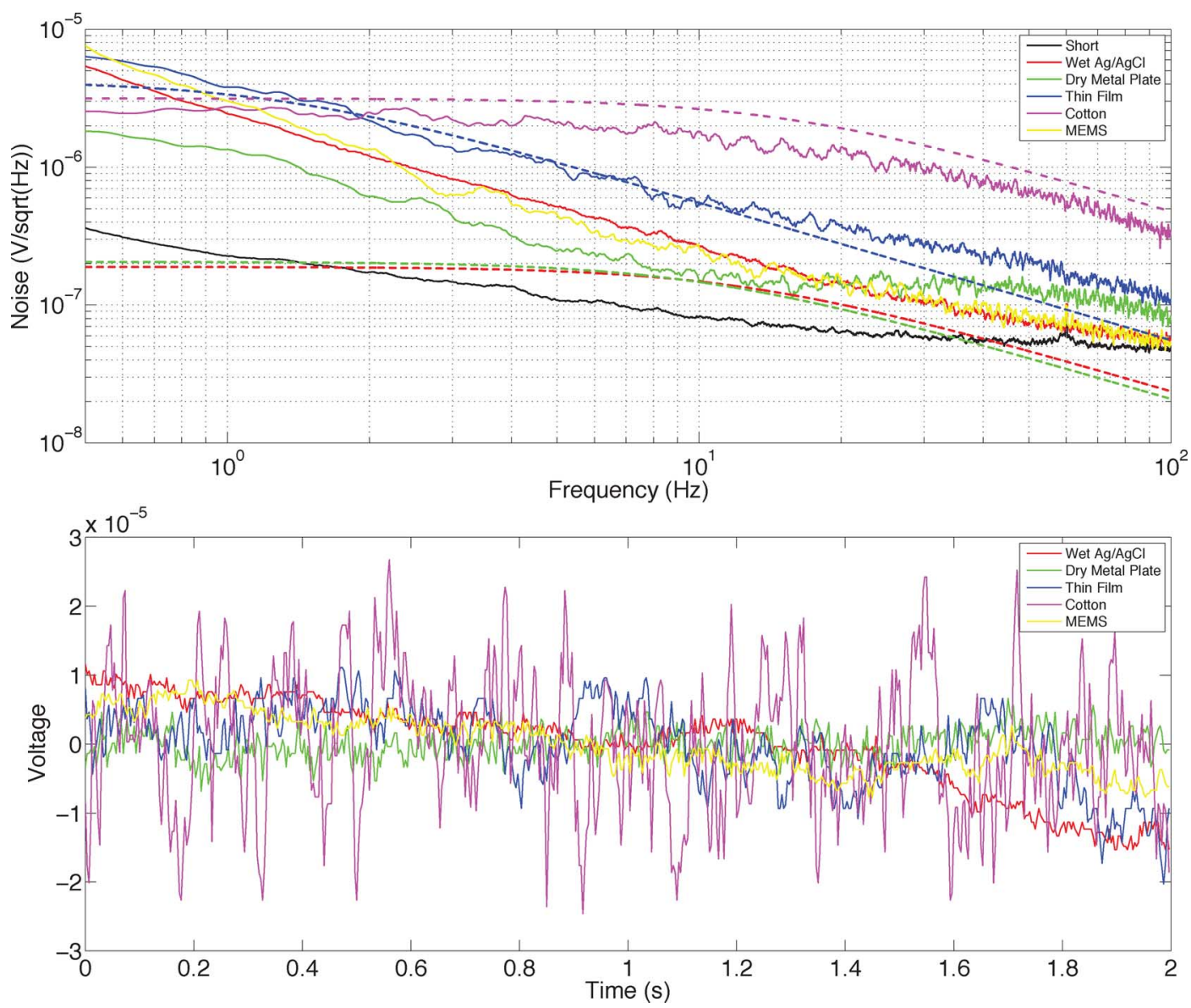

Fig. 4. Measured noise spectrum of various electrode types, placed at close proximity on forearm at rest, along with predicted (dotted lines) thermal noise limits (6) from measured skin-electrode coupling impedance data. (Top) The instrumentation noise floor of the amplifier (Fig. 2) is also shown for reference. (Bottom) Time-domain noise plots are also shown.

These expressions give a quantitative means to analyze the noise performance as well as the motion and friction sensitivity of various electrode topologies in terms of physical and electrical circuit parameters, presented in the following.

\section{B. Noise}

The source input-referred noise power density follows directly from (3) where $v_{i, r m s}^{2}$ and $i_{i, r m s}^{2}$ represent the power (RMS squared) of the two input noise generators, $v_{i, n}$ and $i_{i, n}$

$$
\begin{aligned}
v_{s, r m s}^{2} & =\frac{\left|Y_{c}(j \omega)+Y_{i}(j \omega)+Y_{s}(j \omega)\right|^{2}}{\left|Y_{c}(j \omega)\right|^{2}} v_{i, r m s}^{2}+\frac{i_{i, r m s}^{2}}{\left|Y_{c}(j \omega)\right|^{2}} \\
& =\frac{\left(g_{c}+g_{i}\right)^{2}+\omega^{2}\left(C_{c}+C_{i}+C_{s}\right)^{2}}{g_{c}^{2}+\omega^{2} C_{c}^{2}} v_{i, r m s}^{2}+\frac{i_{i, r m s}^{2}}{g_{c}^{2}+\omega^{2} C_{c}^{2}} .
\end{aligned}
$$

The relative contributions of the two noise components are illustrated in Fig. 3. The first noise component, proportional to the amplifier voltage input noise $v_{i, r m s}^{2}$, is scaled by a factor inversely proportional to the electrode coupling efficiency. For low-impedance contact sensors, this voltage noise component reduces to the amplifier noise floor, while for high-impedance contact sensors such as noncontact geometries, the amplifier voltage noise floor is amplified by a factor $1+\left(C_{i}+C_{s}\right) / C_{c}$. This noise amplification could be reduced by minimizing the active shield capacitance as well as amplifier input capacitance. However, as shown in Fig. 3, this first noise contribution does not typically dominate at frequencies of interest, except for noncontact electrodes at large distance with poor electrode coupling. The second, and typically more significant noise component, is proportional to the net current noise $i_{i, r m s}^{2}$ into the coupling impedance. This net current noise combines thermal noise contributed from the skin-electrode coupling conductance $g_{c}$ and amplifier input conductance $g_{i}$, besides amplifier input current noise $i_{i, n}^{2}$. This noise component is fundamental to the skin-electrode coupling interface which typically dominates contributions from the amplifier. In the limit of a perfect noiseless, infinite input impedance amplifier, the source input-referred noise power density (5) reduces to

$$
v_{s, r m s}^{2} \approx \frac{4 k T g_{c}}{\left|Y_{c}(j \omega)\right|^{2}}=\frac{4 k T}{g_{c}+\frac{\omega^{2} C_{c}^{2}}{g_{c}}} .
$$

Paradoxically, (6) shows that fundamentally the source input-referred noise can be reduced to zero in two limits of particular interest: either infinite coupling conductance (low-resistance contact sensing), or infinite coupling impedance (capacitive noncontact sensing). This presents a rather interesting dichotomy-either of the two extreme cases of zero resistance and infinite resistance of skin-electrode contact are actually optimal for low-noise signal reception. 

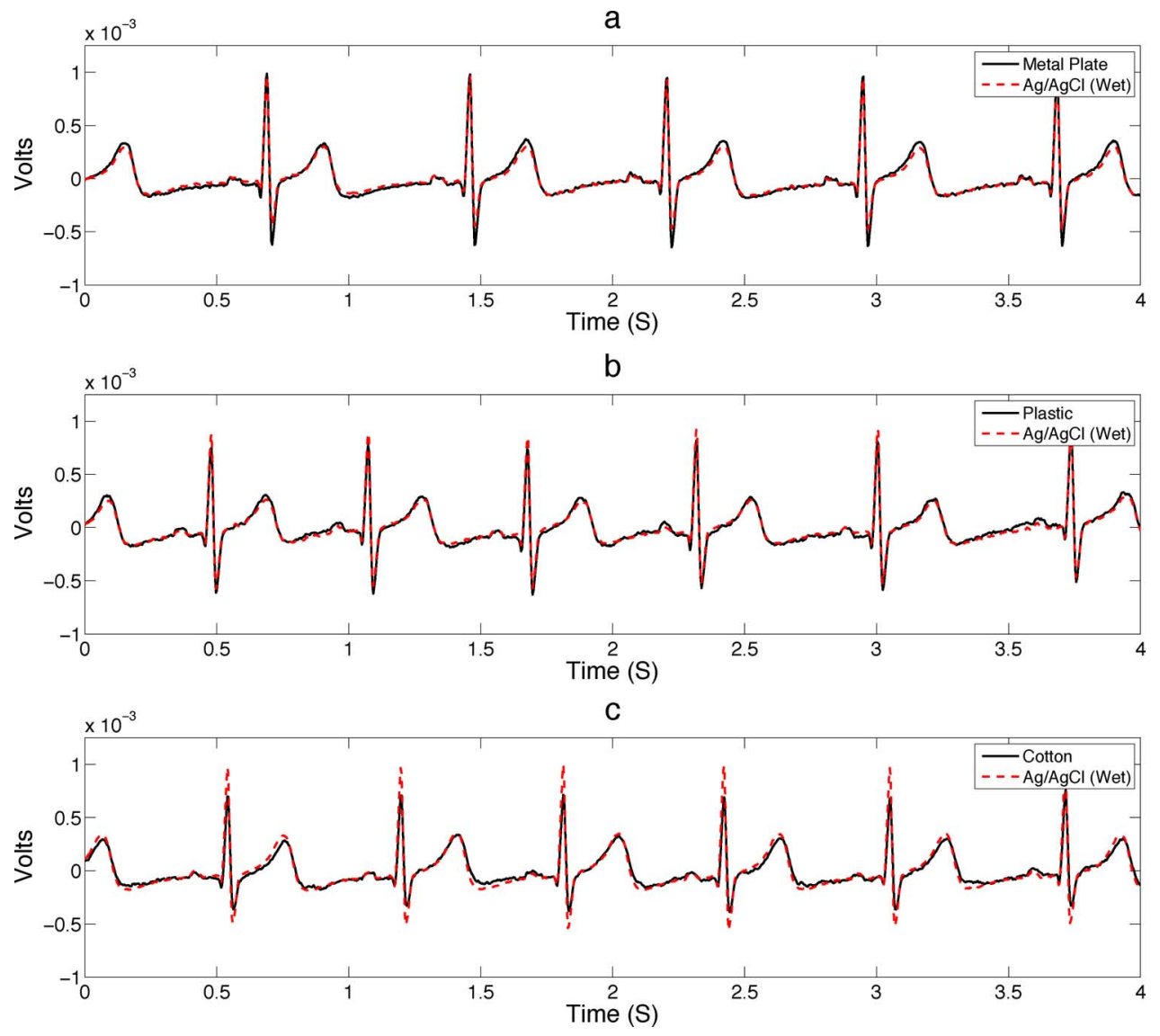

Fig. 5. ECG samples taken from various dry-contact and noncontact test electrodes (metal contact, thin film insulation, cotton noncontact), plotted against signal taken simultaneously from wet $\mathrm{Ag} / \mathrm{AgCl}$ electrode. Data is shown from .7 to $100 \mathrm{~Hz}$ bandwidth without 50/60 Hz notch. Increased noise floor of plastic and cotton electrodes are not readily visible at ECG scales. Signal distortion can be seen on R-wave for cotton electrode due to increased source impedance.

TABLE I

MEASURED ELECTRODE IMPEDANCES

\begin{tabular}{|c||c|}
\hline Wet $\mathrm{Ag} / \mathrm{AgCl}$ & $350 \mathrm{k} \| 25 \mathrm{nF}$ \\
\hline Metal Plate & $1.3 \mathrm{M} \| 12 \mathrm{nF}$ \\
\hline Thin Film & $550 \mathrm{M} \| 220 \mathrm{pF}$ \\
\hline Cotton & $305 \mathrm{M} \| 34 \mathrm{pF}$ \\
\hline MEMS & $650 \mathrm{k} \|$ \\
\hline
\end{tabular}

Measured data on noise obtained from the differential signal between two closely spaced electrodes on the forearm at rest are given in Fig. 4, showing general agreement with the noise model (6) with measured values of coupling resistance and capacitance (Table I). As expected, the instrumentation noise floor of the amplifier (Fig. 2) is dominated by the measured data, confirming that the conditions for the limit model (6) are satisfied. Interestingly, the only electrode type with consistently higher observed noise than the predicted thermal noise from the skin-electrode coupling noise model are the wet-contact $\mathrm{Ag} / \mathrm{AgCl}$ electrodes at lower frequencies. Elevated $1 / f$-like low-frequency drifts of the $\mathrm{Ag} / \mathrm{AgCl}$ offset (half potential mismatch) voltage were confirmed in extended (1-hour) recordings and are consistent with observations in Huigen et al. [4].

One interesting result from this experiment is that for "capacitive" noncontact electrodes operating through clothing [14], [15] , the noise performance and electrode coupling is actually dominated by the resistive component of the cotton layer rather than a capacitance. In many cases, dry contact electrodes are much more capacitively dominated than noncontact electrodes through clothing. Although difficult to imagine, cotton actually acts as a poorly conductive electrode ( $>200 \mathrm{M} \Omega$ ), and is especially harmful for biopotential measurements. The impedance of cotton is such that the coupling is mostly resistive in the frequencies of interest, and amounts to adding a large and noisy series resistor in the signal path. Had the resistance been higher (i.e., very dry), or the shirt been thinner (increased capacitance), the noise floor would have been lower. However, the increased noise did not prevent some acceptable ECG measurements.

Sample ECG data recorded from the same system with metal-plate electrodes mounted on the chest is shown in Fig. 5, showing reasonably accurate correspondence between the dry-contact as well as noncontact electrodes against a wet $\mathrm{Ag} / \mathrm{AgCl}$ electrode reference, even for electrodes placed over a shirt. The capability to continuously record ECG without direct skin contact opens the door to long-term clinical home diagnosis and care applications (Section IV).

\section{Motion and Friction}

Relative motion of electrodes with respect to the body, as well as friction of electrodes against the body surface, give rise to artifacts in the received signals that are one of the main impediments against the acceptance of dry-electrode and noncontact biopotential sensors in mobile clinical settings. These artifacts, 
however, are not unique to electrodes with poor resistive contact and arise in low-resistance wet-contact electrodes as well. They can be reduced, but not eliminated, by partly containing the relative motion to careful mechanical design, although at some expense in the comfort, size and weight of the mounted sensors.

The effect of motion and friction on the signal reception can be readily identified, to first order, from the electrical model (1), (2) and (3). We distinguish between two sources of error that are induced by motion of the electrode relative to the body surface: transversal motion, and lateral motion and friction.

Transversal motion primarily gives rise to instantaneous changes in the skin-electrode coupling impedance, changes which can be discontinuous for contact-based sensors in the absence of a gel bath between skin and electrode. The effect of these impedance changes are similar to the signal arising due to membrane deflections in a microphone and need to be carefully mitigated in the circuit design to avoid vibration and other mechanical deflection sensitivity. According to (2), the effect of changes in coupling admittance $Y_{c}(j \omega)$ are nulled out when the following impedance balancing condition is met:

$$
Y_{i}(j \omega)=j \omega\left(A_{v}-1\right) C_{s}
$$

or, equivalently

$$
\begin{aligned}
g_{i} & =0 \\
C_{i} & =\left(A_{v}-1\right) C_{s} .
\end{aligned}
$$

The zero input conductance condition (8) is readily implemented with a CMOS or other high-impedance amplifier. The balanced capacitance condition (9) is more difficult to implement since input impedance depends on circuit nonidealities that may vary with signal level, such as amplifier protection diodes. The most common approach taken for precise tuning of the capacitive balance is to provide a variable voltage gain $A_{v}$ or trimmed capacitance active shield $C_{c}$, although repeated adjustments may be necessary and are costly to implement. A simple alternative approach, also extensively used, is to provide unity gain active shielding $A_{v} \approx 1$, along with minimizing the input capacitance $C_{i}$. This approach is taken in the active electrode of Fig. 2, with a unity gain connected LMP7702 with $5 \mathrm{pF}$ input capacitance.

Lateral motion in contact may induce friction when the electrode is in direct contact with the skin or with a partially solid coupling medium, a source of error due to possible induction of triboelectric charge onto the electrode surface. No satisfying quantitative models exist to generally describe this effect, but to first order we may consider continuous friction to induce a triboelectric current adding to the net current noise into the amplifier input, resulting in an additional voltage noise component

$$
v_{t, r m s}^{2} \approx \frac{i_{t, r m s}^{2}}{\left|Y_{c}(j \omega)\right|^{2}}=\frac{i_{t, r m s}^{2}}{g_{c}^{2}+\omega^{2} C_{c}^{2}}
$$

which shows that low skin-electrode impedance (either in terms of low coupling resistance, or high coupling capacitance) directly reduces the effect of friction.
Fig. 6 qualitatively illustrates the effect of walking and running body activity, inducing motion and friction in random directions, on the ECG signal recorded using noncontact electrodes over a cotton shirt, in comparison to wet contact sensors simultaneously mounted on the skin under the shirt. A tight vest around the waist assisted in mechanically containing the relative motion, and a wireless interface provided mobility while avoiding common-mode noise and line noise pick-up [14]. The wet contact sensors showed reduced, but not completely eliminated, signal artifacts during activity relative to the noncontact sensors. The $\mathrm{R}$ wave of the ECG however remained clearly visible both for the wet and noncontact sensors. Practical issues with motion and friction are further discussed in Section II-D and more particularly for noncontact sensors in Section III-B.

\section{Practical Design Considerations}

Broadly speaking, two approaches have been taken to resolve the issue of electrode-skin contact impedance for low-noise, low-artifact biopotential sensing. The traditional solution has been to simply abrade the skin to obtain a very low contact resistance $(5-10 \mathrm{k} \Omega)$. At the other extreme, one common practice has been to employ an amplifier with such high input impedance that the skin-electrode impedance becomes negligible. For wet electrodes, neither extreme is necessary, but the problem of contact impedance becomes a much more pressing problem for dry and noncontact sensors, for which maximizing input impedance is the only viable alternative.

Achieving truly nonconductive noncontact sensing, however, is difficult in practice. Fully accounting for the electrical coupling between the skin and the electrode, and its effect on noise (4), is generally quite complex, because of the different layers of coupling involved through skin and the coupling medium (Fig. 1). Low resistance layers generate no appreciable thermal noise. High resistivity layers may generate large thermal noise voltages, but these voltages get shunted away as long as the impedance of the parallel capacitance is sufficiently low over the frequencies of interest. At the most basic level, the coupling impedance can be described as a single resistance in series with a parallel conductance-capacitance combination (center in Fig. 1). In practice, we find (Fig. 4) that all electrode types couple signals both resistively and capacitively in the frequencies of interest for biopotential signals. The interplay between electrode conductance and capacitance is one of the critical factors determining the limits on noise performance.

Also, the success in reducing noise by increasing coupling resistance depends on the impedance level of the coupling capacitance, which strongly depends on frequency. For low capacitive coupling (at large distance), higher electrode resistances translate directly into increased noise levels, both intrinsically due to thermal noise and induced by motion and friction artifacts. According to (6), increasing the coupling resistance only lowers noise for values of resistance $R_{c}$ larger than $1 / \omega C_{c}$. This value becomes exceedingly large for increasing electrode distances. For this reason, the most demanding applications where close proximity to the skin cannot be warranted, like research EEG over haired skull, still require wet electrodes.

In summary, nearly all aspects of the performance of an electrode are critically limited by the physical properties of the inter- 

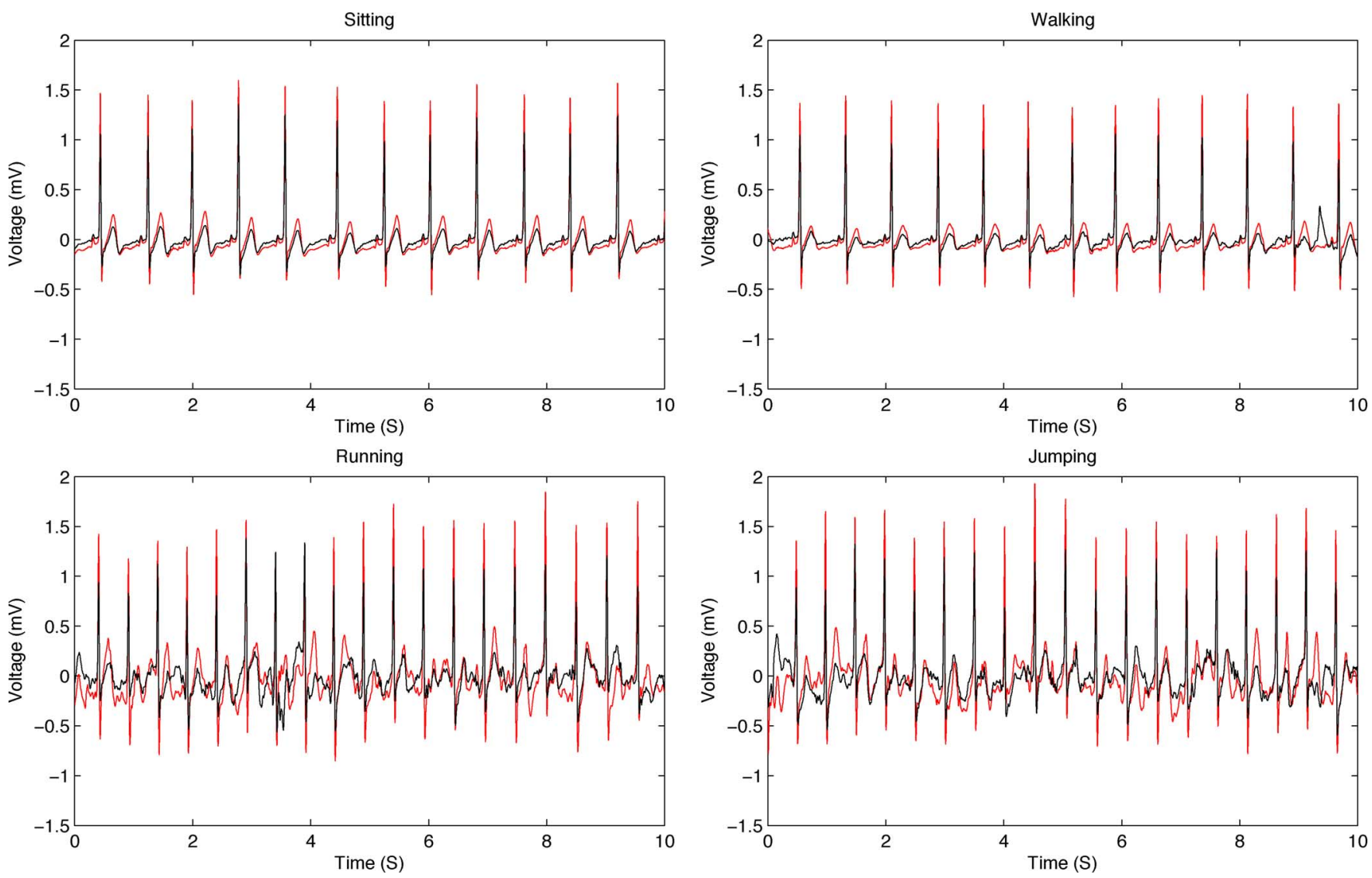

Fig. 6. A 10-s comparison of noise and drift from wet $\mathrm{Ag} / \mathrm{AgCl}$ (red trace) versus noncontact electrodes (black trace) during various activities, inducing motion and friction. Noncontact electrodes were fixed in a tight wireless chest band on top of a cotton shirt [14].

face between skin and the electrode, rather than amplifier goodness criteria (even though these still need to be met).

\section{ELECTRODE TECHNOLOGIES}

\section{A. Dry Electrodes}

In contrast to wet $\mathrm{Ag} / \mathrm{AgCl}$ electrodes, dry electrodes are designed to operate without an explicit electrolyte. Instead, it is usually supplied by moisture on the skin (i.e., sweat). Numerous variations of dry electrodes exist ranging from simple stainless steel discs to micro-fabricated silicon structures with built-in amplifier circuitry. Employing dry contact sensors is somewhat more challenging in practice than traditional techniques largely due to the increased skin-electrode impedance, although the impedance can be quite comparable to wet electrodes after a few minutes due to sweat and moisture buildup [16]. Successful designs use either an active electrode circuit to buffer the signal before driving any cabling or alternatively penetrate the skin to achieve a low contact impedance.

In its simplest form, a dry electrode can be built from any conductive material in contact with the skin, such as a flat metal disc (Fig. 2) and is well-known in the literature [16]. As an example, Valchinov et al. presents a modern variation of this design in 2004. Performance and signal quality of these simple electrodes can be as good as wet electrodes, especially if an amplifier [17] is onboard. Dry electrodes work well for quick measurements (such as exercise machines), but suffer from usability problems for normal clinical applications. Standard wet electrodes usually include an adhesive material to fix the electrode in proper locations, and a hydrogel or wet-foam to both lower the skin impedance, and buffer the electrode against mechanical motion. Adding an adhesive material to place these dry electrode in the proper clinical locations for continuous use eliminates many of its comfort/convenience advantages. Nevertheless, the simplicity and durability of metal dry electrodes make it highly useful for applications like ECG event monitors where short, infrequent use over long periods of time is expected.

Flexible versions of the dry electrode based on rubber [18], fabric [19]-[21] or foam are also possible and more appealing from both a comfort and usability standpoint. Softer materials have the advantage of conforming easily against the skin, increasing comfort and contact area. Gruetzmann et al. demonstrated a foam electrode [22], which exhibited excellent stability with increased resistance to motion artifact versus the wet and rigid dry $\mathrm{Ag} / \mathrm{AgCl}$ electrode.

The high-resistance layer of the skin, the Stratum Corneum, is typically abraded or hydrated to achieve a lower resistance and better electrode contact. It is also possible to penetrate the $10-40-\mu \mathrm{m}$ layer with microfabricated needles [23], [24]. Bypassing the Stratum Corneum can achieve a contact as good as, if not better than, a standard $\mathrm{Ag} / \mathrm{AgCl}$ electrode [23] without the need for any skin preparation or gel. To date, preliminary data has been available for EEG applications of this electrode. However, long-term studies on the hygiene, comfort and safety 
of this technology is unavailable. The authors have observed irritation and slight pain when using these electrodes. It is certainly conceivable that they must be single use, and necessarily be packaged presterilized.

For EEG, recording signals reliably through thick layers of hair remains one of the key challenges. One technique, using dry sensors that do not require scalp preparation, involves the use of thin fingers that can penetrate through hair, first described in a patent by Gevins et al. in 1990 [25]. Several research groups have demonstrated this technique successfully. Matthews et al. [15] presents one well-characterized version of this sensor and shows that the EEG signal obtained can be largely comparable to wet electrodes, for stationary subjects. However, the high skincontact impedance results in a much larger motion artifact with the dry sensors. Fiedler et al. published a TiN-based fingered dry electrode that reported an impedance of $14-55 \mathrm{k} \Omega$ /finger versus around $10 \mathrm{M} \Omega$ /finger [15]).

The final type of dry electrode, first demonstrated by Lopez and Richardson in 1968 [8], does not require ohmic contact at all [10]. In Richardson's original design, a simple Aluminum disc was anodized to form a large blocking capacitor in series with the skin. Signals were capacitively coupled to the input of an FET buffer amplifier and subsequently connected to standard instrumentation.

Taheri et al. expanded on this design by fabricating an insulated electrode on a silicon substrate which integrated a buffer amplifier [26]. It was also designed to have multiple, redundant sensing sites along with a simple algorithm to select the channels that are most likely to have a good contact.

The combination of a good dielectric material combined with physical skin contact means that the coupling capacitance for insulated electrodes is relatively large, from $300 \mathrm{pF}$ [26] to several nanofarads. As a result, designing a bias network with low noise and frequency response for clinical grade signals is very feasible with a standard high-impedance input FET amplifier.

In most respects, the usage and performance of insulated electrodes is quite similar to dry electrodes in practice. Some limited data exists that suggest capacitively coupled electrodes suffer from less skin-motion artifact noise than dry electrodes [3]. More detailed studies need to be conducted to determine what advantage, if any, can be achieved by inserting a layer of insulation between the skin and electrode. From an electrical perspective, the high capacitance of the thin insulation layer is an effective short at signal frequencies and have no effect on the signal quality vis-a-vis dry electrodes. One obvious downside, however, is that the insulated nature of the electrode precludes a frequency response down to DC, which may be important for certain applications.

\section{B. Noncontact, Capacitive Electrodes}

Wet and dry electrodes both require direct physical skin contact to operate. The final type of sensor, the noncontact electrode, can sense signals with an explicit gap between the sensor and body. This enables the sensor to operate without a special dielectric layer and through insulation like hair, clothing or air. Noncontact electrodes have been typically described simply as coupling signals through a small capacitance (10's pF) [11], [12], [27]. In reality, however, there is typically an important resistive element $(>100 \mathrm{M} \Omega$ ) as well, since the typical insulation (i.e., fabric) will also have a non-neglible resistance [28]. As shown previously, signal coupling through noncontact electrodes can be actually dominated by the resistive part of the source impedance which can cause a large input voltage noise.

Designing an amplifier to acquire signals from such a high source impedance is quite challenging. Typical design problems include achieving a high enough input impedance and a stable bias network that does introduce excessive noise. Finally, very high impedance nodes are susceptible to any stray interference and motion induced artifacts.

Nevertheless, in 1994, Prance et al. demonstrated a working noncontact system with an array of 25 ECG senors [29] that was designed to acquire signals with a 3-mm spacing from the body. A low-leakage biasing circuit using a bootstrapped reverse diode, combined with positive feedback to neutralize the parasitic input capacitance, was used to achieve an extremely high impedance, reported at $\left(10^{16} \Omega, 10^{-17} \mathrm{~F}\right)$. However, it is not clear how these measurements were made or over what bandwidth. In addition, the effective input impedance with neutralization is a complex function of both the coupling capacitance and frequency.

In 2000, Prance et al. published an improved version based on the INA116 electrometer instrumentation amplifier from Burr-Brown (Texas Instruments) with a lower noise floor [30]. It again utilizes positive feedback for neutralization of the input capacitance. While the specifics were not published, it can be inferred that the process is far from perfect, as it requires manual calibration and different devices do not match well [31]. Detailed descriptions of bootstrapping and neutralization techniques, however, can be found in unrelated fields [32] as well as a very old publication [33] based on vacuum tubes, but the principles are fully applicable to modern amplifiers. It is not clear as to what advantages of attempting to maintain such a high input impedance are, as many other papers show excellent results with much simpler circuits.

The ability to sense biopotential signals through insulation has resulted in ingenious implementations ranging from sensors mounted on beds [21], [28], chairs [34] and even toilet seats [35]. In general, the signal quality ranges from poor to quite good, as long as proper shielding and subject grounding techniques are utilized [36].

Conventional systems typically use a driven-right-leg active ground to further minimize common-mode noise [37]. Kim et al. makes an important contribution in this field by extending the analysis for the driven-right-leg scheme for capacitive applications [36]. In particular, he shows that an active ground, even capacitively coupled, is highly effective at reducing line noise. It is worthwhile to note that the active ground connection can be capacitive as well for a system that is truly noncontact. This extra degree of common-mode rejection is especially useful in light of the input impedance problem. Alternatively, Matthews et al. reported a proprietary grounding scheme, different from the classical DRL, that employed high-impedance dry electrodes [13].

Unfortunately, specific key circuit and construction details for noncontact sensors have generally not been available in the literature. In particular, the critical information relating to input 


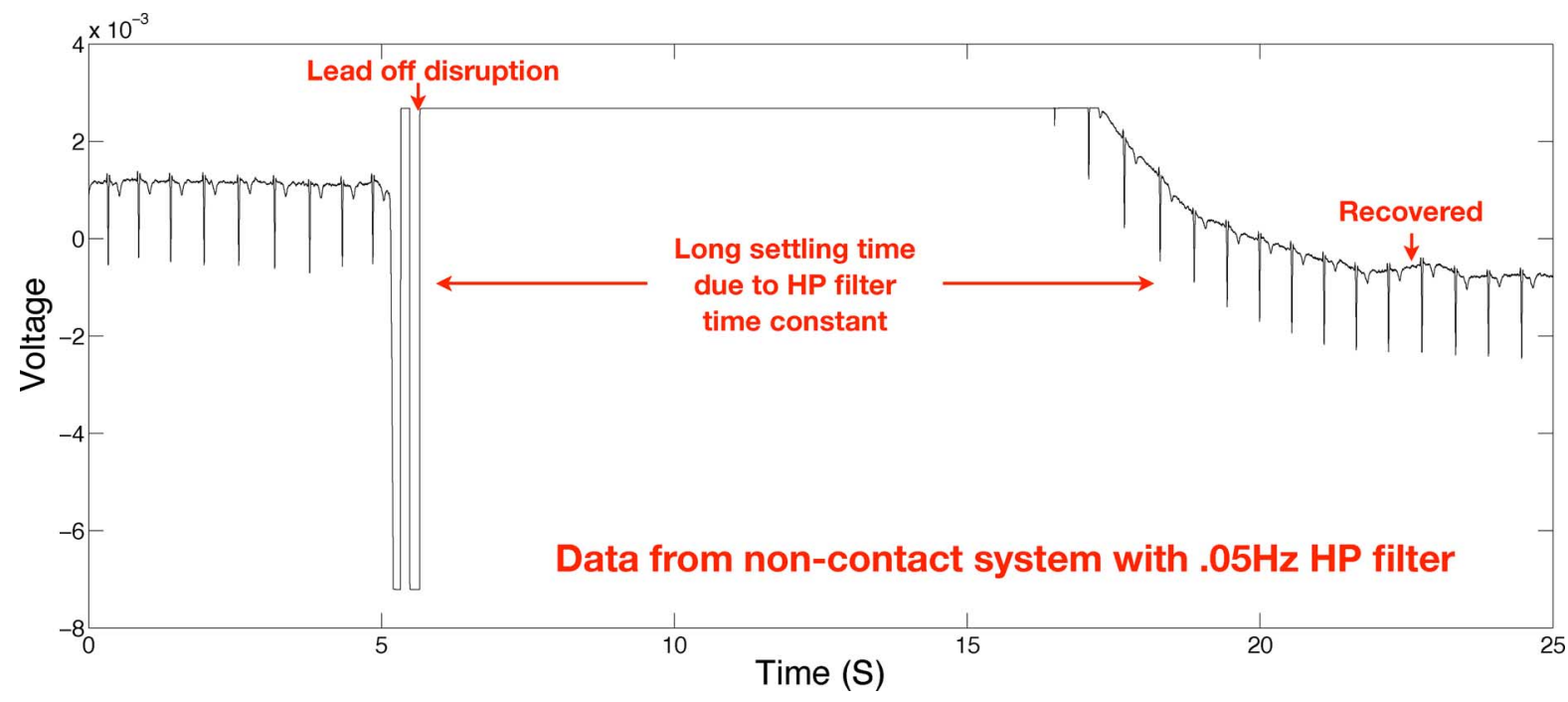

Fig. 7. High impedance input node settling time. Lead was disrupted at $t=5 \mathrm{~s}$. It takes more than $15 \mathrm{~s}$ for trace to recover, showing problem with recovery time for AC coupled instrumentation. Input was designed to have cutoff of $0.05 \mathrm{~Hz}$ in line with ECG standards.

biasing, input capacitance neutralization and circuit reference/ grounding that allow someone to duplicate the sensor and experiments have been scarce. A complete design for a noncontact, wireless ECG/EEG system can be found in [14], which improves and summarizes their previous designs [12], [27], [38], [39]. These noncontact sensor designs are very simple and robust, manufactured completely on a standard PCB with inexpensive and commonly available components (chip resistors, capacitors and the National LMP7723 and LMP2232). The critical input node was left floating and it was found that the input can reliably self-bias purely through the device's internal ESD protection structure and other parasitic leakages. Since no extra conductive devices were added to the input, the circuit achieved the optimal noise performance of the amplifier. The DC offset was simply removed with a passive high-pass filter before the second, differential gain stage. The sensor performed well in laboratory environments and $60 \mathrm{~Hz}$ noise was virtually absent through the use of proper shielding, an active ground and a fully isolated, wireless system. These papers can serve as a useful starting point and timesaver for researchers who wish to develop and experiment with their own noncontact sensors.

One key drawback with capacitive, noncontact sensors, as explained in Section II-C, is their susceptibility to motion artifacts. Several authors have demonstrated performance comparable to clinical adhesive electrodes [13], [14], through a t-shirt, with a moving subject for ECG. The caveat, however, was that this required a tight vest and chest band to secure the noncontact electrodes in place [14]. This highlights the key, unresolved problem with noncontact electrodes-susceptibility to motioninduced artifacts. For noncontact electrodes, artifacts tend to be dominated by three sources. First, the high-impedance, capacitively-coupled, input node of the electrode exhibits a large time settling time constant. Second, displacements in the electrode-to-skin distance can cause artifacts [40]. Finally, friction between the electrode and insulation (fabric, hair, etc.) can cause large voltage excursion at the sensitive input.
Typically, noncontact electrodes exhibit poor settling times due to the high-pass characteristic at the electrode. Fig. 7 shows the settling for a noncontact ECG sensor with a low-frequency response that extends down to $0.05 \mathrm{~Hz}$ prescribed for ECG. Recovery times of upwards of $10 \mathrm{~s}$ can be seen and are exacerbated by the noncontact electrode's susceptibility to movement induced overloads and artifacts. Faster recovery is possible by shifting the corner frequency of the high-pass filter, but at a cost of distorting the signal waveform. Achieving a good frequency response without the settling time problem remains challenging.

All known noncontact sensor designs deliberately limit the high-pass corner frequency to at least around 0.5 to $1 \mathrm{~Hz}$, which introduces appreciable distortion in the ECG waveform. The clinical usefulness of this distorted ECG versus the standard trace is not known by the authors and needs further consideration.

Simple models have been devised to model and solve the displacement artifact for capacitive ECG sensors [40], proposed by Ottenbacher et al., but rely on precise knowledge of the coupling capacitance. Thus, while effective in simulations and controlled bench experiments, it has yet to be reliably demonstrated on actual live recordings. On the other hand, there is no known solution to friction-induced artifacts.

As it stands, there is no real impediment to building fully functional noncontact sensors from standard off-the-shelf amplifiers, and the actual implementation can be as simple as a dry electrode, with proper component selection. For actual usage, the noncontact electrode's susceptibility to motion artifacts, friction and thermal noise are problematic.

\section{SYSTEMS}

The relative utility of dry-contact and noncontact electrodes, in contrast with the more established and widespread wet-contact electrodes, is inextricably tied to novel systems applications or tools that it can enable. In this section, we discuss such enabling systems application domains for two main clinical needs: 


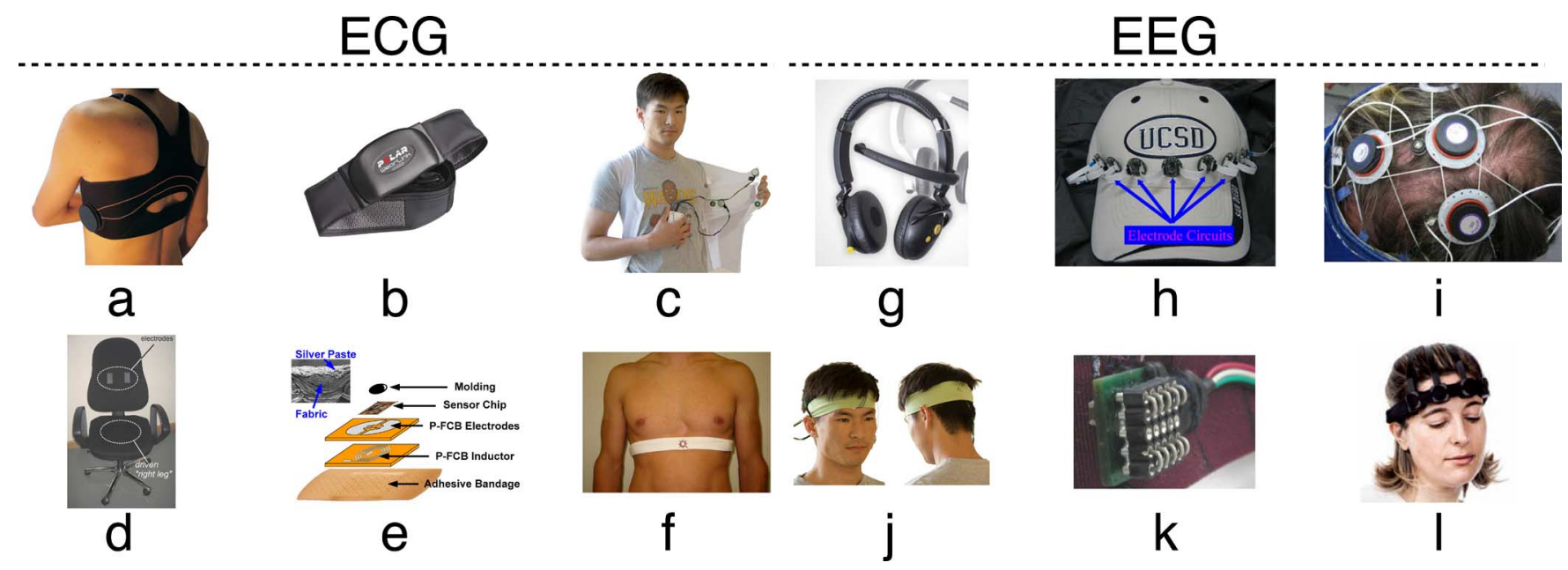

Fig. 8. Dry and noncontact electrode systems. ECG: (a) chest harness [41], (b) polar heart strap, (c) noncontact vest [14], (d) chair [42], [43], (e) wireless band-aid [20] and (f) dry chest strap [15]. EEG: (g) Neurosky single channel headset, (h) dry MEMs cap [44], (i) fingered dry EEG harness [15], (j) dry/noncontact EEG headband [14], (k) dry active electrode [45] and (1) ENOBIO wireless dry sensor.

cardiac and neurological monitoring. Examples of systems in their applications environments for clinical ECG and EEG use are illustrated in Fig. 8.

\section{A. $E C G$}

It is unlikely that dry electrodes will ever replace the adhesive, wet $\mathrm{Ag} / \mathrm{AgCl}$ for in-hospital use. Standard electrodes adhere well to the body, are robust, inexpensive and simple. Properly used, wet electrodes provide an excellent signal. Dry or noncontact electrodes offer few advantages for the majority of hospital applications, while adding cost and complexity (such as the for active electrode circuitry). It is worth noting, however, that for situations where patients with extremely sensitive skin (i.e., burn units [46], neonatal care [47], [48] ), dry and noncontact electrodes may be desirable.

At a basic level, the Polar Heart Rate monitor is one wellknown example, although nonclinical, of a dry electrode based system for cardiac monitoring. The basic theme of a wearable, dry-contact chest strap/harness has been demonstrated by several authors [13], [41] and at least one known medical device company (Monebo). They provide a very easy way to continuously obtain a 1-lead ECG. Given the right analysis and wireless clinical infrastructure, dry-contact chest straps may prove to be a viable tool for long-term cardiac monitoring. With noncontact sensors, it is also possible to build a strap/harness that can be worn on top of a t-shirt [14], with electrodes placed in approximate positions to provide a derived 12-lead ECG [14]. Motion artifacts and chest tightness, however, remain a difficulty with wearable, noncontact systems.

Small bandage-like patches are even more convenient than chest straps for long-term, mobile monitoring. Recent advancements in microelectronics electronics have made it possible to integrate an entire ECG monitoring system within a small patch. Yoo et al. presents an inductively powered ECG chest patch [20], [49], [50] based on a single integrated circuit mounted on a fabric substrate. A few commercial offerings are also now on the market, in a somewhat larger form-factor
(Corventis, iRythm, Proteus). Unfortunately, the short electrode-to-electrode distance makes it impossible to obtain the same waveform as even a standard 1-lead ECG, although the QRS complex is readily visible in the most cases. These devices have the potential to be highly useful for basic long-term cardiac monitoring, such as arrhythmia detection.

Besides mobile wearable devices, noncontact electrodes have been used for rapidly obtaining chest body surface potential maps (BSPM). In fact, the first demonstration of noncontact electrodes [29] was for a chest array. Newer versions have been developed, mounted on a standard tablet PC [51]. Noncontact electrodes have a distinct advantage since they can be taken through clothing without any preparation. However, it is not clear what the clinical advantages are for noncontact BSPMs, especially in light of the noise and frequency responses of noncontact electrodes. A contact version, perhaps embedded within a tight garment, could prove useful, provided the extra information over a 12-lead ECG is clinically relevant.

Clinical ECG monitoring devices have traditionally required patients to wear a device on the body. With the exception of an implantable monitor, all of these systems require some degree of patient intervention and compliance. The advent of noncontact electrode technology has made it possible to integrate cardiac monitoring devices unobtrusively in the environment. Several attempts have been made to integrate electrodes in beds [28], chairs [34], [43], [52] and even bathtubs [53] and toilets [35]. Obtaining signs of cardiac activity through an air gap $(40 \mathrm{~cm})$ is also possible [54]. Unfortunately, signal quality from these devices is typically quite poor and riddled with motion artifact, noise and interference problems. At present, nothing has progressed beyond a basic proof-of-concept. More detailed clinical studies are required to find out if the degree of monitoring provided by beds and chairs is clinically useful.

\section{B. $E E G$}

Unlike ECG, which has a long and established clinical practice of outpatient monitoring systems, the difficulty in preparing 


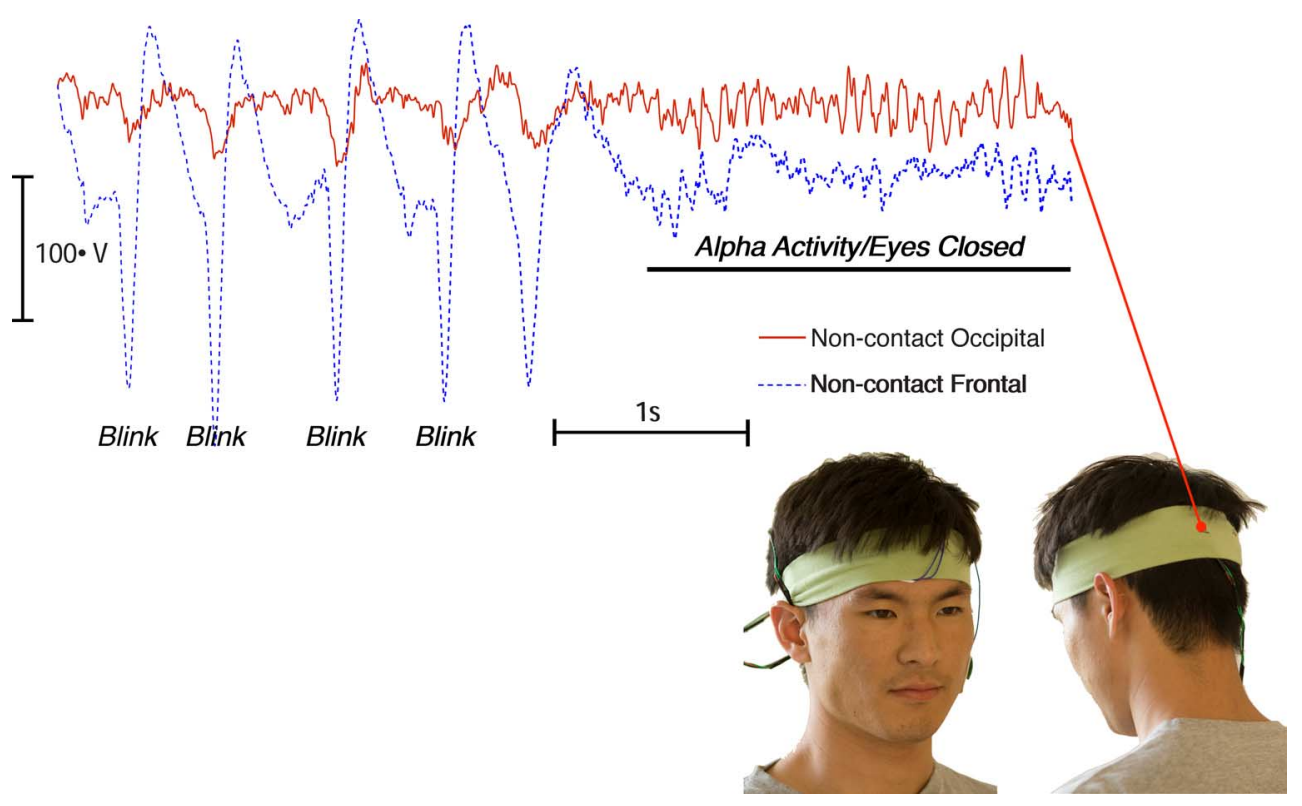

Fig. 9. Noncontact EEG headband and data from both frontal and occipital electrodes [14].

a patient and data interpretation has largely limited brain monitoring to in-hospital settings. With the exception of an EEG counter part to the 24/48-hour ECG Holter device, mobile clinical EEG devices are still rarely used today. However, there does exist a strong need and interest for EEG monitoring for medical conditions such as sleep apnea, epilepsy and traumatic brain injury. Thus, novel applications and uses are even more critical for the success of dry electrode technology for EEG. Thus, it is expected that these wireless, outpatient EEG-based neural monitoring systems will become much more commonplace in the near future. A robust and patient-friendly dry electrode system will be a significant contribution to this field.

At this time, there exists no clinical dry/noncontact EEG device on the market. Several commercial offerings have been made mostly with a consumer focus for entertainment (Neurosky), sleep/wellness (Zeo) and marketing (Emsense). However, there has been significant activity with using dry EEG systems for research use [15], [44], [55].

Sullivan et al. presented an architecture for high-density, dryelectrode EEG based around the concept of integrating the entire signal processing (amplification, filtering, digitizing) chain on top of a dry MEMS electrode [44]. This enables electrodes to be easily daisy-chained and expanded with only one common wire, significantly reducing the clutter associated with a conventional EEG system. It is easily wearable and provides access to the forehead locations without gels or other preparation.

Monitoring of user attention or alertness is another area that has been explored as a candidate for dry-contact EEG. Several headsets have been developed with this in mind [15], [45]. In 2009, Tsai et al. presented a detailed study of using dry-contact EEG sensors to monitor for driver drowsiness [45].

Fig. 9 shows a prototype dry EEG system that was able to acquire signals from the back of the head, albeit with a very tight band. Successfully obtaining signals from dry hair, without preparation, is an ongoing area of development for EEG systems.

\section{CONCLUSION}

From an electronics perspective, almost all of the circuit design issues are now well understood and well described in literature, from achieving high common-mode rejection to building micropower circuitry. In essence, a modern FET-input amplifier configured in unity-gain will be more than sufficient to buffer signals from virtually any electrode. Achieving a sufficiently high input impedance is not a problem for the majority of cases. Input offsets are problematic, but DC-coupled instrumentation with very low gains $(0 \mathrm{~dB})$ and high-resolution ADCs (24-bit) can tolerate large electrode offsets. Except for esoteric applications, such as ECG sensing through a large air-gap, it is unlikely any circuit innovation directly at the electrode will be highly useful. It goes without saying, however, that there is much room for circuit/electronics innovation at the system level for building integrated, wearable and wireless biopotential sensors.

Resolving the difficulties with motion artifacts remains the unsolved challenge in mobile, wearable ECG/EEG sensor systems. Unlike circuit characterization which involves standard, easily simulated and readily measured parameters like noise, gain and power consumption, motion artifacts are ill-defined and subject to human variability. In addition, different types of electrodes suffer from artifacts from distinct sources. The lack of quantifiable merits compounded with the difficulty in obtaining measurements has resulted in less attention in this area.

In addition, fully understanding and characterizing the origin of skin-electrode noise is another under-addressed area in this field. It is well known that the noise level, while strongly correlated with skin impedance, far exceeds the amount predicted by thermal noise at low frequencies [4]. It has been theorized that the redox reaction at the electrode accounts for the $1 / f$ characteristic with wet $\mathrm{Ag} / \mathrm{AgCl}$ electrodes [4]. It has not been established that electrochemical noise contributes to capacitively coupled noncontact sensors, since redox reactions do not take place across the interface. Our theory and experimental 
observations have shown that for noncontact electrodes, the thermal noise model is more accurate and provides some clear guidelines for design considerations in the electrode interface. Again the lack of standard measurement methods combined with human variability makes an objective comparison scarce and difficult. An establishment of a clear measurement protocol followed by detailed, objective, comparisons of the noise behavior of all electrode types will be highly illuminating.

Overall, there also needs to be a greater emphasis on the materials, packaging, signal processing and systems level. The ultimate solution will likely be a combination of some circuit design, but even more a matter of innovative mechanical construction and signal processing. Efforts directed in that direction are expected to yield significant returns for this field.

\section{APPENDIX \\ ELECTRICAL NOISE MODEL}

From the definition of voltage gain and input referred voltage noise of the amplifier, ignoring dynamics, the output voltage is given by

$$
v_{o}=A_{v}\left(v_{i}+v_{i, n}\right) .
$$

Similarly, KCL at the input node of the amplifier in the diagram of Fig. 4 yields

$$
Y_{c}(j \omega)\left(v_{s}-v_{i}\right)-Y_{i}(j \omega) v_{i}+j \omega C_{s}\left(v_{o}-v_{i}\right)+i_{i, n}=0 .
$$

Elimination of $v_{i}$ from (11) and (12) leads to

$$
\begin{aligned}
& \left(Y_{c}+Y_{i}+j \omega C_{s}-A_{v} j \omega C_{s}\right) v_{o}= \\
& \quad A_{v}\left(Y_{c} v_{s}+\left(Y_{c}+Y_{i}+j \omega C_{s}\right) v_{i, n}+i_{i, n}\right)
\end{aligned}
$$

which together with $Y_{c}(j \omega)=g_{c}+j \omega C_{c}$ and $Y_{i}(j \omega)=g_{i}+$ $j \omega C_{i}$ retrieves the electrical model (1) with signal voltage gain (2) and source input-referred voltage noise (3).

\section{ACKNOWLEDGMENT}

The authors would like to thank Dr. C. Maier for his many helpful insights and discussions. Copyright (c) 2010 IEEE. Personal use of this material is permitted. However, permission to use this material for any other purposes must be obtained from the IEEE by sending an email to pubs-permissions@ieee.org.

\section{REFERENCES}

[1] X.-F. Teng, Y.-T. Zhang, C. Poon, and P. Bonato, "Wearable medical systems for p-health," IEEE Rev. Biomed. Eng., vol. 1, no. 1, pp. 62-74, Jan. 2008.

[2] A. Lymberis, "Smart wearable systems for personalised health management: Current R and D and future challenges," in Proc. IEEE 25th Annu. Int. Conf. Eng. Medicine Biology Soc., 17-21, 2003, vol. 4, pp. 3716-3719.

[3] A. Searle and L. Kirkup, "A direct comparison of wet, dry and insulating bioelectric recording electrodes," Physiological Measure., vol. 21, no. 2, p. 271, 2000.

[4] E. Huigen, A. Peper, and C. A. Grimbergen, "Investigation into the origin of the noise of surface electrodes," Med. Biological Eng. Comput., vol. 40, no. 3, pp. 332-338, 2002.
[5] M. Spach, R. Barr, J. Havstad, and E. Long, "Skin-electrode impedance and its effect on recording cardiac potentials," Circulation, vol. 34, pp. 649-656, 1966.

[6] A. Baba and M. Burke, "Measurement of the electrical properties of ungelled ECG electrodes," Int. J. Biol. Biomed. Eng., vol. 2, no. 3, pp. 89-97, 2008.

[7] J. Bourland, L. Geddes, G. Sewell, R. Baker, and J. Kruer, "Active cables for use with dry electrodes for electrocardiography," J. Electrocardiol., vol. 11, no. 1, pp. 71-74, 1978.

[8] A. Lopez and P. C. Richardson, "Capacitive electrocardiographic and bioelectric electrodes," IEEE Trans. Biomed. Eng., vol. BME-16, no. 1, pp. 99-99, Jan. 1969.

[9] B. A. Taheri, R. T. Knight, and R. L. Smith, "A dry electrode for EEG recording," Electroencephalography Clin. Neurophysiol., vol. 90, no. 5, pp. 376-383, 1994.

[10] T. Matsuo, K. Iinuma, and M. Esashi, "A barium-titanate-ceramics capacitive-type EEG electrode," IEEE Trans. Biomed. Eng., vol. BME-20, no. 4, pp. 299-300, Jul. 1973.

[11] C. J. Harland, T. D. Clark, and R. J. Prance, "Electric potential probes-New directions in the remote sensing of the human body," Measure. Sci. Technol., vol. 13, no. 2, p. 163, 2002.

[12] T. Sullivan, S. Deiss, and G. Cauwenberghs, "A low-noise, non-contact EEG/ECG sensor," in Proc. IEEE Biomedical Circuits Systems Conf., 2007, pp. 154-157.

[13] R. Matthews, N. McDonald, P. Hervieux, P. Turner, and M. Steindorf, "A wearable physiological sensor suite for unobtrusive monitoring of physiological and cognitive state," in Proc. IEEE Annu. Int. Conf. Engineering Medicine Biology Soc., 2007, pp. 5276-5281.

[14] Y. M. Chi, P. Ng, E. Kang, J. Kang, J. Fang, and G. Cauwenberghs, Wireless Health, Oct. 2010.

[15] R. Matthews, N. J. McDonald, H. Anumula, J. Woodward, P. J. Turner, M. A. Steindorf, K. Chang, and J. M. Pendleton, "Novel hybrid bioelectrodes for ambulatory zero-prep eeg measurements using multi-channel wireless EEG system," in Proc. 3rd Int. Conf. Foundations Augmented Cognition, Berlin, Germany, 2007, pp. 137-146.

[16] L. Geddes and M. Valentinuzzi, "Temporal changes in electrode impedance while recording the electrocardiogram with "dry" electrodes," Ann. Biomed. Eng., vol. 1, pp. 356-367, 1973.

[17] E. Valchinov and N. Pallikarakis, "An active electrode for biopotential recording from small localized bio-sources," BioMed. Eng. OnLine, vol. 3, no. 1, p. 25, 2004

[18] G. Gargiulo, P. Bifulco, R. Calvo, M. Cesarelli, C. Jin, and A. v. Schaik, "Mobile biomedical sensing with dry electrodes," in Proc. Int. Conf. Intelligent Sensors, Sensor Networks Information Processing, ISSNIP, 2008, pp. 261-266.

[19] M. Mestrovic, R. Helmer, L. Kyratzis, and D. Kumar, "Preliminary study of dry knitted fabric electrodes for physiological monitoring," in Proc. 3rd Int. Conf. Intelligent Sensors, Sensor Networks Information, 2007, pp. 601-606.

[20] J. Yoo, L. Yan, S. Lee, H. Kim, B. Kim, and H.-J. Yoo, "An attachable ECG sensor bandage with planar-fashionable circuit board," in Proc. Int. Symp. Wearable Computers ISWC, 2009, pp. 145-146.

[21] M. Ishijima, "Monitoring of electrocardiograms in bed without utilizing body surface electrodes," IEEE Trans. Biomed. Eng., vol. 40, no. 6, pp. 593-594, Jun. 1993.

[22] A. Gruetzmann, S. Hansen, and J. Müller, "Novel dry electrodes for ECG monitoring," Physiol. Measure., vol. 28, no. 11, p. 1375, 2007.

[23] P. Griss, P. Enoksson, H. Tolvanen-Laakso, P. Merilainen, S. Ollmar, and G. Stemme, "Spiked biopotential electrodes," in Proc. 13th Annu. Int. Conf. Micro Electro Mechanical Systems MEMS, 2000, pp. 323-328

[24] C. Chang and J. Chiou, "Surface-mounted dry electrode and analogfront-end systems for physiological signal measurements," in Proc. IEEE/NIH Life Science Systems Applic. Workshop, LiSSA, 2009, pp. $108-111$.

[25] A. S. Gevins, D. Durousseau, and J. Libove, "Dry electrode brain wave recording system," Patent U.S. 4967 038, Oct. 30, 1990.

[26] B. Alizadeh-Taheri, R. L. Smith, and R. T. Knight, "An active, microfabricated, scalp electrode array for EEG recording," Sensors Actuators A: Physical, vol. 54, no. 1-3, pp. 606-611, 1996.

[27] Y. Chi and G. Cauwenberghs, "Micropower non-contact EEG electrode with active common-mode noise suppression and input capacitance cancellation," in Proc. Annu. Int. Conf. Eng. Medicine Biol. Soc., EMBC, 2009, pp. 4218-4221. 
[28] A. Ueno, Y. Akabane, T. Kato, H. Hoshino, S. Kataoka, and Y. Ishiyama, "Capacitive sensing of electrocardiographic potential through cloth from the dorsal surface of the body in a supine position: A preliminary study," IEEE Trans. Biomed. Eng., vol. 54, no. 4, pp. 759-766, Apr. 2007.

[29] A. Clippingdale, R. J. Clark, and C. Watkins, "Ultrahigh impedance capacitively coupled heart imaging array," Rev. Scientific Instruments, vol. 65, no. 1, pp. 269-270, 1994.

[30] R. J. Prance, A. Debray, T. D. Clark, H. Prance, M. Nock, C. J. Harland, and A. J. Clippingdale, "An ultra-low-noise electrical-potential probe for human-body scanning," Measure. Sci. Technol., vol. 11, no. 3, p. 291, 2000.

[31] W. Gebrial, R. J. Prance, C. J. Harland, and T. D. Clark, "Noninvasive imaging using an array of electric potential sensors," Rev. Scientific Instruments, vol. 77, no. 6, p. 063708, 2006.

[32] A. Saab and R. White, "Achieve femtoampere leakage in surface-mount op-amp layouts," Electron. Design, Strategy, News, Jul. 2008.

[33] C. Guld, "Cathode follower and negative capacitance as high input impedance circuits," Proc. IRE, vol. 50, no. 9, pp. 1912-1927, Sep. 1962.

[34] A. Aleksandrowicz and S. Leonhardt, "Wireless and non-contact ECG measurement system-The Aachen SmartChair," ActaPolytechnica, vol. 2, pp. 68-71, Jun. 2007.

[35] K. K. Kim, Y. K. Lim, and K. S. Park, "The electrically noncontacting ecg measurement on the toilet seat using the capacitively-coupled insulated electrodes," in Proc. 26th Annu. Int. Conf. IEEE Eng. Medicine Biol. Soc., 2004, vol. 1, pp. 2375-2378.

[36] K. K. Kim and K. S. Park, "Effective coupling impedance for power line interference in capacitive-coupled ecg measurement system," in Proc. Int. Conf. Information Technology Applic. Biomedicine ITAB, 2008, pp. 256-258.

[37] B. B. Winter and J. G. Webster, "Driven-right-leg circuit design," IEEE Trans. Biomed. Eng., vol. BME-30, no. 1, pp. 62-66, Jan. 1983.

[38] Y. M. Chi and G. Cauwenberghs, "Wireless non-contact EEG/ECG electrodes for body sensor networks," in Proc. Int. Conf. Body Sensor Networks (BSN), 2010, pp. 297-301.

[39] Y. Chi, S. Deiss, and G. Cauwenberghs, "Non-contact low power EEG/ECG electrode for high density wearable biopotential sensor networks," in Proc. 6th Int. Workshop Wearable Implantable Body Sensor Networks BSN, 3-5, 2009, pp. 246-250.

[40] J. Ottenbacher and S. Heuer, II, "Motion artefacts in capacitively coupled ECG electrodes," IFMBE Proc., vol. 25, no. 4, pp. 1059-1062, 2009.

[41] S. Fuhrhop, S. Lamparth, and S. Heuer, "A textile integrated long-term ECG monitor with capacitively coupled electrodes," in Proc. IEEE Biomedical Circuits Systems Conf. BioCAS, 2009, pp. 21-24.

[42] M. Steffen, A. Aleksandrowicz, and S. Leonhardt, "Mobile noncontact monitoring of heart and lung activity," IEEE Trans. Biomed. Circuits Syst., vol. 1, no. 4, pp. 250-257, Dec. 2007.

[43] K. K. Kim, Y. K. Lim, and K. S. Park, "Common mode noise cancellation for electrically non-contact ECG measurement system on a chair," in Proc. 27th Annu. Int. Conf. Engineering Medicine Biol. Soc. IEEE-EMBS, 2005, pp. 5881-5883.

[44] T. Sullivan, S. Deiss, T.-P. Jung, and G. Cauwenberghs, "A brain-machine interface using dry-contact, low-noise EEG sensors," in Proc. IEEE Int. Symp. Circuits Systems ISCAS, 2008, pp. 1986-1989.

[45] P.-Y. Tsai, W. Hu, T. Kuo, and L.-Y. Shyu, "A portable device for real time drowsiness detection using novel active dry electrode system," in Proc. IEEE Int. Conf. Engineering Medicine Biol. Soc. EMBC, 2009, pp. 3775-3778.

[46] M. Griffith, W. Portnoy, L. Stotts, and J. Day, "Improved capacitive electrocardiogram electrodes for burn applications," Med. Biol. Eng. Comput., vol. 17, no. 5, pp. 641-646, 1979.

[47] S. Bouwstra, L. Feijs, W. Chen, and S. Oetomo, "Smart jacket design for neonatal monitoring with wearable sensors," in Proc. 6th Int. Int. Workshop Wearable Implantable Body Sensor Networks BSN, 2009, pp. 162-167.

[48] T. Kato, A. Ueno, S. Kataoka, H. Hoshino, and Y. Ishiyama, "An application of capacitive electrode for detecting electrocardiogram of neonates and infants," in Proc. 28th Annu. Int. Conf. Eng. Medicine Biol. Soc. EMBS, Aug. 2006, pp. 916-919.

[49] J. Yoo, L. Yan, S. Lee, H. Kim, and H.-J. Yoo, "A wearable ECG acquisition system with compact planar-fashionable circuit board-based shirt," IEEE Trans. Inform. Technol. Biomedicine, vol. 13, no. 6, pp. 897-902, Nov. 2009.
[50] J. Yoo, L. Yan, S. Lee, Y. Kim, and H.-J. Yoo, “A 5.2 mw self-configured wearable body sensor network controller and a $12 \mathrm{w}$ wirelessly powered sensor for a continuous health monitoring system," IEEE J. Solid-State Circuits, vol. 45, no. 1, pp. 178-188, Jan. 2010.

[51] M. Oehler, V. Ling, K. Melhorn, and M. Schilling, "A multichannel portable ECG system with capacitive sensors," Physiological Measure., vol. 29, no. 7, p. 783, 2008.

[52] Y. G. Lim, K. K. Kim, and S. Park, "ECG measurement on a chair without conductive contact," IEEE Trans. Biomed. Eng., vol. 53, no. 5, pp. 956-959, May 2006.

[53] Y. K. Lim, K. K. Kim, and K. S. Park, "The ECG measurement in the bathtub using the insulated electrodes," in Proc. 26th Annu. Int. Conf. Engineering Medicine Biol. Soc. IEMBS, 1-5, 2004, vol. 1, pp. 2383-2385.

[54] R. J. Prance, S. T. Beardsmore-Rust, P. Watson, C. J. Harland, and H. Prance, "Remote detection of human electrophysiological signals using electric potential sensors," Appl. Phys. Lett., vol. 93, no. 3, p. 033906, 2008.

[55] G. Ruffini, S. Dunne, E. Farres, P. Watts, E. Mendoza, S. Silva, C. Grau, J. Marco-Pallares, L. Fuentemilla, and B. Vandecasteele, "Enobio-First tests of a dry electrophysiology electrode using carbon nanotubes," in Proc. 28th Annu. Int. Conf. Engineering Medicine Biol. Soc. EMBS, Aug. 2006, pp. 1826-1829.

[56] K.-I. Yamakoshi, "In the spotlight: Bioinstrumentation," IEEE Rev. Biomed. Eng., vol. 2, pp. 2-5, 2009.

[57] T. Sullivan, S. Deiss, and G. Cauwenberghs, "A low-noise, non-contact EEG/ECG sensor," in Proc. IEEE Biomedical Circuits Systems Conf. BIOCAS, 2007, pp. 154-157.

[58] T. Maruyama, M. Makikawa, N. Shiozawa, and Y. Fujiwara, "ECG measurement using capacitive coupling electrodes for man-machine emotional communication," in Proc. IEEE/ICME Int. Conf. Complex Medical Eng., 2007, pp. 378-383.

[59] C. Park, P. Chou, Y. Bai, R. Matthews, and A. Hibbs, "An ultra-wearable, wireless, low power ECG monitoring system," in Proc. IEEE Biomedical Circuits Systems Conf., Nov. 2006, pp. 241-244.

[60] J.-C. Chiou, L.-W. Ko, C.-T. Lin, C.-T. Hong, T.-P. Jung, S.-F. Liang, and J.-L. Jeng, "Using novel MEMS EEG sensors in detecting drowsiness application," in Proc. IEEE Biomedical Circuits Systems Conf. BioCAS, Nov. 2006, pp. 33-36.

[61] C.-T. Lin, L.-W. Ko, J.-C. Chiou, J.-R. Duann, R.-S. Huang, S.-F. Liang, T.-W. Chiu, and T.-P. Jung, "Noninvasive neural prostheses using mobile and wireless EEG," Proc. IEEE, vol. 96, no. 7, pp. 1167-1183, Jul. 2008.

[62] J. M. Lee, F. Pearce, C. Morrissette, and A. Hibbs, "Evaluating a capacitively coupled, noncontact electrode for ECG monitoring," Sensors Mag., 2005.

[63] A. P. A. C. MettingVanRijn and C. Grimbergen, "Amplifiers for bioelectric events: A design with a minimal number of parts," Med. Biological Eng. Comput., 1994.

[64] C. J. Harland, T. D. Clark, N. S. Peters, M. J. Everitt, and P. B. Stiffell, "A compact electric potential sensor array for the acquisition and reconstruction of the 7-lead electrocardiogram without electrical charge contact with the skin," Physiological Measure., vol. 26, no. 6, p. 939, 2005.

[65] J. Rosell, J. Colominas, P. Riu, R. Pallas-Areny, and J. Webster, "Skin impedance from $1 \mathrm{~Hz}$ to $1 \mathrm{mHz}$," IEEE Trans. Biomed. Eng., vol. 35, no. 8, pp. 649-651, Aug. 1988.

[66] H. J. Scheer, T. Sander, and L. Trahms, "The influence of amplifier, interface and biological noise on signal quality in high-resolution EEG recordings," Physiological Measure., vol. 27, no. 2, p. 109, 2006.

[67] A. Hassibi, R. Navid, R. W. Dutton, and T. H. Lee, "Comprehensive study of noise processes in electrode electrolyte interfaces," J. Appl. Phys., vol. 96, no. 2, pp. 1074-1082, 2004.

[68] B. Eggins, "Skin-electrode impedance and its effect on recording cardiac potentials," Analyst, vol. 118, pp. 439-442, 1993.

[69] M. Puurtinen, J. Viik, and J. Hyttinen, "Best electrode locations for a small bipolar ECG device: Signal strength analysis of clinical data," Ann. Biomed. Eng., vol. 37, no. 2, pp. 331-336, 2009.

[70] T. C. Ferree, P. Luu, G. S. Russell, and D. M. Tucker, "Scalp electrode impedance, infection risk, and EEG data quality," Clinical Neurophysiol. : Official J. Int. Federation Clinical Neurophysiol., vol. 112, no. 3, pp. 536-544, Mar. 2001.

[71] J. Muhlsteff, O. Such, R. Schmidt, M. Perkuhn, H. Reiter, J. Lauter, J. Thijs, G. Musch, and M. Harris, "Wearable approach for continuous ECG and activity patient-monitoring," in Proc. IEEE 26th Annu. Int. Conf. Eng. Medicine Biology Soc. IEMBS, 2004, vol. 1, pp. 2184-2187. 
[72] P. Fiedler, S. Bordkorb, C. Fonseca, F. Vaz, F. Zanow, and J. Haueisen, "Novel TiN-based dry eeg electrodes; influence of electrode shape and number on contact impedance and signal quality," in IFMBE Proc., May 2010, vol. 29, pp. 418-421.

[73] P. Tallgren, S. Vanhatalo, K. Kaila, and J. Voipio, "Evaluation of commercially available electrodes and gels for recording of slow EEG potentials," Clin. Neurophysiol., vol. 116, no. 4, pp. 799-806, 2005.

[74] C. Fonseca, J. Cunha, R. Martins, V. Ferreira, J. de Sa, M. Barbosa, and A. da Silva, "A novel dry active electrode for EEG recording," IEEE Trans. Biomed. Eng., vol. 54, no. 1, pp. 162-165, Jan. 2007.

[75] G. Ruffini, S. Dunne, E. Farres, I. Cester, P. Watts, S. Ravi, P. Silva, C. Grau, L. Fuentemilla, J. Marco-Pallares, and B. Vandecasteele, "Enobio dry electrophysiology electrode; First human trial plus wireless electrode system," in Proc. IEEE 29th Annu. Int. Conf. Eng. Medicine Biol. Soc. EMBS, 2007, pp. 6689-6693.

[76] C. Assambo and M. Burke, "Amplifier input impedance in dry electrode ECG recording," in Proc. IEEE Annu. Int. Conf. Eng. Medicine Biol. Soc. EMBC, 2009, pp. 1774-1777.

[77] K.-P. Hoffmann and R. Ruff, "Flexible dry surface-electrodes for ECG long-term monitoring," in Proc. 29th Annu. Int. Conf. Eng. Medicine Biol. Soc. EMBS, 2007, pp. 5739-5742.

[78] B. He and R. Cohen, "Body surface laplacian ECG mapping," IEEE Trans. Biomed. Eng., vol. 39, no. 11, pp. 1179-1191, Nov. 1992.

[79] N. Verma, A. Shoeb, J. Bohorquez, J. Dawson, J. Guttag, and A. Chandrakasan, "A micro-power EEG acquisition SOC with integrated feature extraction processor for a chronic seizure detection system," IEEE J. Solid-State Circuits, vol. 45, no. 4, pp. 804-816, Apr. 2010.

[80] J.-Y. Baek, J.-H. An, J.-M. Choi, K.-S. Park, and S.-H. Lee, "Flexible polymeric dry electrodes for the long-term monitoring of ECG," Sensors Actuators A: Physical, vol. 143, no. 2, pp. 423-429, 2008.

[81] P. Griss, H. Tolvanen-Laakso, P. Merilainen, and G. Stemme, "Characterization of micromachined spiked biopotential electrodes," IEEE Trans. Biomed. Eng., vol. 49, no. 6, pp. 597-604, Jun. 2002.

[82] J. Ottenbacher, L. Jatoba, U. Grossmann, W. Stork, and K. MüllerGlaser, "ECG electrodes for a context-aware cardiac permanent monitoring system," IFMBE Proc., vol. 14, no. 7, pp. 672-675, 2007.

[83] L. Fay, V. Misra, and R. Sarpeshkar, "A micropower electrocardiogram amplifier," IEEE Trans. Biomed. Circuits Syst., vol. 3, no. 5, pp. 312-320, Oct. 2009.

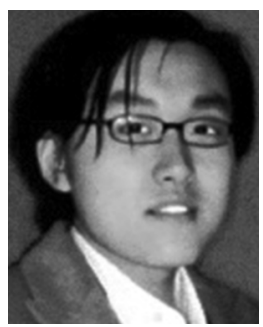

Yu Mike Chi (S'09) received the B.S. degree in electrical engineering from the Johns Hopkins University, Baltimore, MD, and the M.S. degree in electrical engineering from the University of California, San Diego. He is currently working toward the Ph.D. degree at the same university.

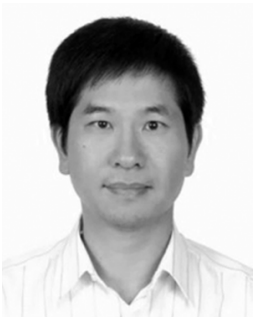

Tzyy-Ping Jung (SM'98) received the B.S. degree in electronics engineering from National Chiao-Tung University, Taiwan, R.O.C., in 1984, and the M.S. and $\mathrm{Ph} . \mathrm{D}$. degrees in electrical engineering from The Ohio State University, Columbus, in 1989 and 1993 , respectively.

He was a Research Associate with the Computational Neurobiology Laboratory, The Salk Institute, La Jolla, CA. He is currently a Research Scientist with the Institute for Neural Computation and Associate Director of the Swartz Center for Computational Neuroscience, University of California, San Diego. He is also a Professor in the Department of Computer Science, National Chiao Tung University, Hsinchu, Taiwan. His research interests are in the areas of biomedical signal processing, cognitive neuroscience, machine learning, time-frequency analysis of human EEG, functional neuroimaging, and braincomputer interfaces and interactions.

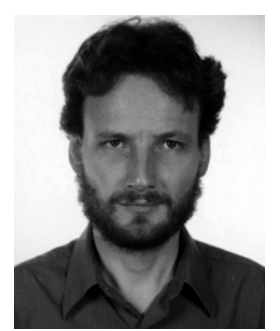

Gert Cauwenberghs (SM'01) received the Ph.D. degree in electrical engineering from the California Institute of Technology, Pasadena.

Currently, he is a Professor of Bioengineering at the University of California, San Diego, where he codirects the Institute for Neural Computation. Previously, he was a Professor of electrical and computer engineering at Johns Hopkins University, Baltimore, MD, and Visiting Professor of brain and cognitive science at the Massachusetts Institute of Technology, Cambridge. His research interests cover neuromorphic engineering, computational and systems neuroscience, neuron-silicon and brain-machine interfaces, as well as learning and intelligent systems.

Dr. Cauwenberghs is an Associate Editor for IEEE TRANSACTIONS ON BIOMEDICAL CIRCUITS AND SYSTEMS, and IEEE TRANSACTIONS ON NEURAL SYSTEMS AND REHABILITATION ENGINEERING. He is a Senior Editor for the IEEE SENSORS JOURNAL. He received a National Science Foundation Career Award in 1997, an ONR Young Investigator Award in 1999, and Presidential Early Career Award for Scientists and Engineers in 2000. 